\title{
The prevalence of social phobia among students of Rafsanjan University of Medical Sciences, Iran, and its relation with personality traits in 2013
}

\author{
Agha Mohammad Hasani P, MD ${ }^{1}$, Mokhtaree MR, MSc ${ }^{2 *}$, Asadollahi Z, MSc ${ }^{3}$, Fereidoni MJ, \\ Medical Student ${ }^{4}$
}

1- Assistant Prof., Dept. of Psychiatry, Rafsanjan University of Medical Sciences, Rafsanjan, Iran. 2- Msc of Educational Psychology, Rafsanjan University of Medical Sciences, Rafsanjan, Iran. 3- Faculty Member, Dept. of Epidemiology and Biostatistics, Rafsanjan University of Medical Sciences, Rafsanjan, Iran. 4- Medical Student, Rafsanjan University of Medical Sciences, Rafsanjan, Iran

\begin{abstract}
Received: July 2016, Accepted: October 2016

Background: Social phobia is a pervasive and debilitating disorder, with relatively high prevalence, which is affected by different variables and influences many aspects of human life. This study was carried out with the aim to determine the relationship between social anxiety and personality dimensions among students of Rafsanjan University of Medical Sciences, Iran, in 2013.

Materials and Methods: The study population included all students of Rafsanjan University of Medical Sciences in 2013. The sample volume was estimated utilizing Cochran's formula as 284 individuals. The participants were chosen through stratified random sampling method from among the statistical population. Participants answered the Social Phobia Inventory (SPIN) designed by Connor et al., which evaluates social phobia and its components that are fear, avoidance, and physiological signs, and the NEO Personality Inventory (NEO PI-R) which evaluates five personality traits. The data were analyzed using the Pearson correlation, ANOVA, independent t-test, and multivariate regression.

Results: The prevalence of social phobia among the subjects, except in mild cases, was $58.5 \%$, of which, $19.4 \%$ suffered from severe social phobia and $10.2 \%$ from very severe social phobia. Among personality dimensions, neuroticism had a direct meaningful relation with social phobia and its aspects. In addition, extraversion, agreeableness, openness to experience, and conscientiousness were inversely associated with social phobia. The personality dimensions of neuroticism and openness to experience were effective predictors of social phobia.

Conclusions: The results are indicative of the high prevalence of social phobia among medical students. They also suggest that social phobia and its dimensions have a direct meaningful relation with neuroticism personality dimension. Neuroticism and agreeableness personality dimensions had the necessary power to predict social phobia.
\end{abstract}

Keywords: Social Phobia, Personality, Medical, Student

\section{Introduction}

Social phobia is a type of anxiety identified by through evident and persistent fear, and severe anxiety in social situations or functions and it can impair one's daily activities (1). Social phobia is a disabling disorder that affects many aspects of a person's life, and in extreme cases, significantly reduces the individual's quality of life (QOL). Many people suffering from this disorder may not leave the house for a long period of time or lose a many social positions such as occupational and educational

\footnotetext{
* Corresponding author: Mohammadreza Mokhtaree, Rafsanjan University of Medical Sciences, Rafsanjan, Iran. E-mail: mrmokhtaree@yahoo.com
} 
opportunities. The ratio of the prevalence of this disease among men and women is 2 to 3 , and about $3 \%$ to $13 \%$ of people have the social phobia criteria at some point in their lives (2). Social phobia has the third highest prevalence after major depressive disorder and alcohol addiction (3). Based on the Diagnostic and Statistical Manual of Mental Disorders, Fifth Edition, (DSM-5), the prevalence of social phobia in adolescents is reported as $1 \%$ to $3 \%$, and about $27 \%$ to $47 \%$ of adolescents have reported at least one symptom of this disorder (4).

The somatic symptoms of this disorder include blushing, excessive sweating, tremor, palpitations, nausea, and slurred speech. In cases of severe panic and anxiety, panic attacks may also occur. Early diagnosis and intervention in this disorder can significantly reduce symptoms and slow its progression, and prevent complications such as severe depression. Some people suffering from social phobia use alcohol or drugs and psychedelic substances to reduce fear, increase their confidence, and improve social functioning (5).

Many people are not aware of this disorder, and they think it is derived from personality problems; as a result, they do not search for an appropriate treatment of this disorder. Another factor that is effective in preventing people from going to a doctor is the disorder itself. These issues increase the possibility of addiction to drugs and psychotropic substances, and dangerous drug interactions. It is possible to treat a person suffering from this disorder through medical procedures, psychotherapy, or both. The root of this anxiety is in childhood when human personality is formed.

The prevalence of social phobia differs in different parts of the world and different cultures. For example, the prevalence of social phobia has been reported as about $10 \%, 11.7 \%$, $10 \%, 5.2 \%, 3.9 \%$, and $10.6 \%$ in Indian (6), Saudi Arabian (7), British (8), and Ugandan adolescents (9), Turkish students (10), and Swedish adolescents (11). In addition, the prevalence of social phobia among Brazilian (12), Turkish (13), Swedish (14), and Nigerian students (15) has been reported as $11.2 \%$, $20.9 \%, 16.1 \%$, and $8.5 \%$. Previous studies have provided conflicting reports regarding the prevalence of this disease in Iran. Mazhari (16), Yaghobi (17), Momeni (18), and Bahrami (19) have reported its prevalence among students as $40 \%, 13.2 \%, 28.6 \%$, and 20\%. Numerous studies have examined social phobia and its relationship with psychological variables and structures. Mazhari did not find a relationship between social phobia and academic performance, but he indicated that the academic performance of students, due to the phobia of speaking in public, is significantly lower than their actual ability (16). Moreover, Savari observed a significant negative correlation between social phobia and social competence (20). Bayani reported that students with higher levels of social phobia have poor problemsolving ability and greater depression (20). It has also been shown that social phobia is correlated with adjustment problems (21), intolerance of ambiguities (22), mood disorders (23), increase in cigarette and marijuana use (24), social isolation (25), QOL (26), avoidant personality $(27,28)$, and bipolar disorder (29).

Personality and psychopathology are two categories of the field of psychology, which have gained the interest of many researchers. Several different studies in this field have shown correlations between subsets of these two categories. It seems that personality, as a fundamental psychological construct, is related with several psychiatric disorders (5). Personality and its features are the main concepts in psychology (30), and personality psychologists have presented different definitions of personality. Allport stated: "Personality is a dynamic organization within the individual of those psychophysical systems 
that determine his characteristic behavior and thought".

Rogers also defines personality as a permanent organized self at the center of all human experiences. Watson believes that "personality is an organized series of habits." Clay sees personality as equivalent to each individual's unique way of attempting to interpret life. McCrae and John have described personality in terms of five dimensions or five main factors including neuroticism, extraversion, and openness to experience, agreeableness, and conscientiousness (31).

As stated above, social phobia is correlated with different variables. However, the question is what fundamental variables affect social phobia and what relationship it has with them. For example, can social phobia be affected by a person's personality streaks? Few studies have studied the relation between general personality traits and social phobia. Furthermore, the studies conducted in this field have predominantly focused on the relation between avoidant personality and social phobia $(14,27,28,32-$ 34). On the other hand, a requisite of some medical professions such as medicine and nursing is extensive and direct communication and even lectures in classrooms and communities. Therefore, students suffering from social phobia will have many problems in this area, which will ultimately lead to academic and professional problems (35). Hence, considering the importance of this issue, this study was conducted in the year 2013 with the aim to determine the prevalence of social phobia among students of Rafsanjan University of Medical Sciences, Iran, and its relationship with personality traits.

\section{Materials and Methods}

The study population of this descriptive study consisted of all students studying at Rafsanjan University of Medical Sciences in the fields of medicine, dentistry, nursing, midwifery, anesthesia, operating room, laboratory, radiology, and medical emergency in the year 2013. The participants were stratified into three categories of medicine, dentistry, and other medical majors. The sample size was calculated based on Cochran's formula as 284 individuals. The list of students was obtained from the Department of Education, and 284 participants were selected through stratified random sampling method from the list. After visiting the workplace (colleges or hospitals) or living location (dormitories) of the selected individuals, explanations about the purpose of the study, how to complete the questionnaires, and confidentiality of information were presented to participants. Participants who were willing to take part in the study were enrolled. Then, the Social Phobia Inventory (SPIN) designed by Connor et al. and NEO Personality Inventory (NEO PI-R) (Persian versions) were distributed among them and collected after one hour. Finally, data in questionnaires were encoded and analyzed using SPSS software (version 18, SPSS Inc., Chicago, IL, USA). Quantitative data were presented as mean \pm standard deviation and qualitative data as number and percentage. For data analysis, chisquared test, the Pearson correlation, independent t-test, analysis of variance (ANOVA), and multiple regression were used. Level of significance was considered as 0.05 .

Social Phobia Inventory: The SPIN was prepared for the first time in 2000 by Connor et al. to evaluate this component. The SPIN is a self-report paper and pencil questionnaire with 17 items and 3 subscales including fear, avoidance, and physiological symptoms. The items are scored based on a Likert scale ranging from 0 ("Not at all") to 4 ("Extremely"). Therefore, the minimum score on this scale is 0 and the maximum is 68. A higher score on this scale represents greater social phobia. Based on the standard z-scores, the severity of symptoms based on subjects' scores are described with five 
scales consisting of no observable symptoms (percentile rank of less than 20), mild (percentile rank of 20-40), moderate (percentile rank of 4170 ), severe (percentile rank of 71-90), and very severe symptoms (percentile rank of 90 and higher). Thus, a score of 0 to 29, 30 to 35,36 to 42,43 to 58 , and higher than 58 will, respectively, signify no observable symptoms, and mild, moderate, severe, and very severe symptoms. The cut-off point of the scale was obtained as 12 with $90 \%$ sensitivity and $88 \%$ specificity.

The reliability of this scale, using Cronbach's alpha, was obtained as 0.79 for the total sample, 0.8 for girls, and 0.79 for boys (36). In the present study, Cronbach's alpha of the SPIN was 0.89 for the total sample, 0.898 for girls, and 0.88 for boys.

NEO Personality Inventory: The NEO PI-R was designed by Costa and McCrae in 1985 and it was revised in 1989. The NEO PI-R includes 60 items and measures five personality factors. Items are scored based on a Likert scale ranging from 4 ("Strongly Disagree") to 0 ("Strongly Agree"). Every 12 items measure one of the big five personality factors (neuroticism, extroversion, openness to experience, agreeableness, and conscientiousness). The score of each subscale ranges from 0 to 48 . This questionnaire contains 5 subscales of neuroticism, extraversion, openness to experience, agreeableness, and conscientiousness. Respondents who have higher scores in the neuroticism subscale are more likely to have irrational beliefs, be less able to control their impulses, and show weaker degrees of compatibility with others and adaptability to stressful conditions. Individuals who obtain higher scores on the extraversion subscale are social, decisive, active, and talkative, like to communicate, and love others. Respondents who score high on the openness to experience subscale are curious about the world and enriching their inner experiences, and their life is filled with various experiences. The agreeableness, subscale focuses on interpersonal communication trends. An agreeable person is altruist, sympathizes with others and is willing to help them, and believes that others have the same relationship with him/her. The conscientiousness subscales has two major features; the ability to control momentums and desires, and the implementation of plans in behavior for achieving goals. Individuals with a high score in this subscale have preset goals and strong demands. They are accurate, reliable, and punctual. People with low scores on this subscale do not have sufficient precision to perform tasks, and they are not persistent in reaching goals. The validity coefficients $(0.75-$ 0.83 ) of this questionnaire were calculated within a sample of students within 3 months. The long-term reliability coefficient (6 years) for neuroticism, extraversion, and openness to experience subscales ranged from 0.68 to 0.83 , and for agreeableness and conscientiousness subscales (with an interval of 2 years) have been reported, respectively, as 0.79 and $0.63(37,38)$.

\section{Results}

In this study, 284 individuals were studied, of whom, $54.6 \%$ were women with an average age of $21.3 \pm 2.3$ years, and $45.4 \%$ were men were with an average age of $21.8 \pm 2$ years. Moreover, $71.1 \%$ of the studied individuals were single. In terms of prevalence of academic majors, $33.5 \%$ were medical students, $14.4 \%$ were dental students, and $52.1 \%$ were students of other medical majors.

The average score of social phobia and its dimensions were compared between men and women, and single and married individuals. The average scores of social phobia and all of its dimensions (fear, avoidance, and physiological symptoms) were higher in men than women; however, this difference was not statistically significant $(\mathrm{P}=0.33, \mathrm{P}=0.93, \mathrm{P}=0.18$, and $\mathrm{P}=$ 0.24 , respectively). In addition, there was no 
difference between single and married individuals in terms of social phobia $(\mathrm{P}=0.84)$ and its dimensions including fear $(\mathrm{P}=0.87)$, avoidance $(\mathrm{P}=0.62)$, and physiological symptoms $(\mathrm{P}=0.79)$ (Table 1$)$. ANOVA results indicated that there was no significant difference between the different academic majors in terms of social phobia $(\mathrm{P}=0.22)$, avoidance dimension $(\mathrm{P}=0.58)$, and physiological symptoms $(\mathrm{P}=$ 0.43 ). Nevertheless, the average score of fear (an aspect of social phobia) of dental students (15.68 \pm 5.1) was significantly higher than medical students $(15.01 \pm 4.32)$ and other medical specialties $(13.75 \pm 5.17)(\mathrm{P}=0.034)$.

Table 1: Group differences in social phobia and its dimensions between different gender and marital status groups

\begin{tabular}{|c|c|c|c|c|c|c|}
\hline \multirow{2}{*}{ Variable } & Woman & Man & \multirow{2}{*}{ P-Value } & Single & Married & \multirow{2}{*}{ P-Value } \\
\hline & \multicolumn{2}{|c|}{ Mean \pm SD } & & \multicolumn{2}{|c|}{ Mean \pm SD } & \\
\hline Social Phobia & $38.93 \pm 12.7$ & $40.35 \pm 11.7$ & $\mathrm{P}=0.33$ & $39.67 \pm 12.47$ & $39.34 \pm 11.82$ & $\mathrm{P}=0.84$ \\
\hline Fear & $14.42 \pm 5.15$ & $14.48 \pm 4.69$ & $\mathrm{P}=0.93$ & $14.48 \pm 4.9$ & $14.38 \pm 5.04$ & $\mathrm{P}=0.87$ \\
\hline Avoidance & $16.53 \pm 5.66$ & $17.39 \pm 4.87$ & $\mathrm{P}=0.18$ & $17.02 \pm 5.59$ & $16.67 \pm 4.61$ & $\mathrm{P}=0.62$ \\
\hline Physiological symptoms & $7.98 \pm 3.16$ & $8.48 \pm 3.81$ & $\mathrm{P}=0.24$ & $8.17 \pm 3.41$ & $8.29 \pm 3.63$ & $\mathrm{P}=0.79$ \\
\hline
\end{tabular}

Furthermore, the prevalence of social phobia was also investigated. Among 284 individuals, $21.1 \%$ were without fear, $20.4 \%$ had mild social phobia, $28.9 \%$ suffered from moderate social phobia, $19.4 \%$ suffered from severe social phobia, and $10.2 \%$ had very severe social phobia. The different severities of social phobia in terms of gender, marital status, and academic major were investigated. The results of the present study showed that $11.6 \%$ of men and $9 \%$ of women had very severe social phobia. Chisquared test results showed a significant relationship between the severity of social phobia and gender $(\mathrm{P}=0.012)$. In addition, $8.5 \%$ of single individuals and $10.2 \%$ of married individuals suffered from very sever social phobia; however, the relationship between social phobia and marital status was not significant $(\mathrm{P}=0.45)$. Finally, the prevalence of very severe social phobia was $8.4 \%$ among medical students, $7.3 \%$ among dental students, and $12.2 \%$ among students of other medical specialties. Furthermore, $58.9 \%$ of the 95 studied medical students had moderate to very severe social phobia, $57.4 \%$ of the 148 students from other medical specialties suffered from moderate to severe social phobia, and $60.9 \%$ of the 41 dental students suffered from moderate to severe social phobia. Chi-squared test results showed a significant relation between the severities of social phobia and academic major $(\mathrm{P}=0.009)$ (Table 2).

Table 2: Distribution of different severities of social phobia in terms of gender, marital status, and academic major

\begin{tabular}{|c|c|c|c|c|c|c|c|c|c|c|}
\hline \multirow[t]{2}{*}{ Social phobia } & Man & Woman & Total & Single & Married & Total & Medical & Dental & $\begin{array}{c}\text { Other } \\
\text { medical } \\
\text { specialties }\end{array}$ & Total \\
\hline & $\begin{array}{c}\text { Number } \\
(\%)\end{array}$ & $\begin{array}{c}\text { Number } \\
(\%)\end{array}$ & $\begin{array}{c}\text { Number } \\
(\%)\end{array}$ & $\begin{array}{c}\text { Number } \\
(\%)\end{array}$ & $\begin{array}{c}\text { Number } \\
(\%)\end{array}$ & $\begin{array}{c}\text { Number } \\
(\%)\end{array}$ & $\begin{array}{c}\text { Number } \\
(\%)\end{array}$ & $\begin{array}{c}\text { Number } \\
(\%)\end{array}$ & $\begin{array}{c}\text { Number } \\
(\%)\end{array}$ & $\begin{array}{c}\text { Number } \\
(\%)\end{array}$ \\
\hline $\begin{array}{c}\text { Without } \\
\text { symptoms }\end{array}$ & $18(14)$ & $42(27.1)$ & $60(21.1)$ & $44(21.8)$ & $16(19.5)$ & $60(21.1)$ & $14(14.7)$ & $9(22)$ & $37(25)$ & $60(21.1)$ \\
\hline Mild symptoms & $36(27.9)$ & $22(14.2)$ & $58(20.4)$ & $36(17.8)$ & $22(26.8)$ & $58(20.4)$ & $25(26.3)$ & $7(17.1)$ & $26(17.6)$ & $58(20.4)$ \\
\hline $\begin{array}{l}\text { Moderate } \\
\text { symptoms }\end{array}$ & $36(27.9)$ & $46(29.7)$ & $82(28.9)$ & $62(30.7)$ & $20(24.4)$ & $82(28.9)$ & $24(25.3)$ & $8(19.5)$ & $50(33.8)$ & $82(28.9)$ \\
\hline $\begin{array}{c}\text { Severe } \\
\text { symptoms }\end{array}$ & $24(18.6)$ & $31(20)$ & $55(19.4)$ & $38(18.8)$ & $17(20.7)$ & $55(19.4)$ & $24(25.3)$ & $14(34.1)$ & $17(11.5)$ & $55(19.4)$ \\
\hline $\begin{array}{l}\text { Very severe } \\
\text { symptoms }\end{array}$ & $15(11.6)$ & $14(9.0)$ & $29(10.2)$ & $22(10.9)$ & $7(8.5)$ & $29(10.2)$ & $8(8.4)$ & $3(7.3)$ & $18(12.2)$ & $29(10.2)$ \\
\hline Total & $129(100)$ & $155(100)$ & $284(100)$ & $202(100)$ & $82(100)$ & $284(100)$ & $95(100)$ & $41(100)$ & $148(100)$ & $248(100)$ \\
\hline Chi-squared test & \multicolumn{3}{|c|}{$\mathrm{X}=20.5, \mathrm{df}=8, \mathrm{p}=0.009$} & \multicolumn{4}{|c|}{$\mathrm{X}=3.69, \mathrm{df}=4, \mathrm{p}=0.45$} & \multicolumn{3}{|c|}{$\mathrm{X}=12.85, \mathrm{df}=4, \mathrm{p}=0.012$} \\
\hline
\end{tabular}


There was no difference between women and men in terms of neuroticism $(\mathrm{P}=0.16)$, openness to experience $(\mathrm{P}=0.19)$, and agreeableness $(\mathrm{P}=$ 0.44). However, the average score of extroversion $(\mathrm{P}<0.001)$ and conscientiousness $(\mathrm{P}$ $=0.031)$ in men were significantly higher than in women (Table 3). The results of ANOVA showed that among the five main personality dimensions, average score of conscientiousness among medical students (16.67 \pm 5.9$)$ was significantly higher than among dental students $(14.83 \pm 7.1)$ and other medical specialties $(14.85 \pm 5.4)(\mathrm{P}=0.046)$.

Table 3: Group differences in personality dimensions between different gender and marital status groups

\begin{tabular}{|c|c|c|c|c|c|}
\hline $\begin{array}{ll}\text { Gender } & \text { Variable } \\
\end{array}$ & Neuroticism & Extraversion & $\begin{array}{l}\text { Openness to } \\
\text { experience }\end{array}$ & Agreeableness & Conscientiousness \\
\hline Man & $24.32 \pm 6.58$ & $19.05 \pm 5.78$ & $22.3 \pm 5.3$ & $19.09 \pm 6.18$ & $16.28 \pm 6.24$ \\
\hline Woman & $25.46 \pm 6.99$ & $16.64 \pm 4.61$ & $23.1 \pm 50.3$ & $19.64 \pm 5.73$ & $14.77 \pm 5.48$ \\
\hline Significance level & $\mathrm{P}=0.16$ & $\mathrm{P}<0.001$ & $\mathrm{P}=0.19$ & $\mathrm{P}=0.44$ & $\mathrm{P}=0.031$ \\
\hline
\end{tabular}

In the next step, the relationship between personality dimensions and social phobia was examined. Neuroticism personality dimension had a direct and significant correlation with social phobia and all of its aspects (fear, avoidance, and physiological symptoms) $(\mathrm{P}<0.001)$. Extraversion personality dimension had an inverse significant relationship with social phobia $(\mathrm{P}=0.002)$, fear, and avoidance $(\mathrm{P}=0.001)$ (Table 4).

Table 4: The correlation coefficients of personality dimensions with social phobia and its dimensions

\begin{tabular}{ccccc}
\hline $\begin{array}{l}\text { Personality } \\
\text { dimensions }\end{array}$ & Social Phobia & Foer & Avoidance & Physiological signs \\
\hline Neuroticism & $0.43^{* *}$ & $0.47^{* *}$ & $0.32^{* *}$ & $0.36^{* *}$ \\
\hline Extraversion & $-0.18^{* *}$ & $-0.22^{* *}$ & $-0.23^{* *}$ & 0.02 \\
\hline Openness to experience & $-0.16^{* *}$ & -0.09 & $0.21^{* *}$ & -0.1 \\
\hline Agreeableness & $-0.23^{* *}$ & $-0.2^{* *}$ & $-0.27^{* *}$ & $-0.13^{*}$ \\
\hline Conscientiousness & $-0.19^{* *}$ & $-0.19^{* *}$ & $-0.2^{* *}$ & $-0.12^{*}$ \\
\hline
\end{tabular}

Multiple regression was used to predict social phobia in terms of personality dimensions. The regression equation was significant $(\mathrm{P}<0.001)$, and $23 \%$ of variance in social phobia could be explained by personality dimensions. Data analysis showed that neuroticism and openness to experience with beta coefficients of 0.424 and -0.217 , respectively, were effective in social phobia prediction, which means that these two variables could significantly predict social phobia $(\mathrm{P}<0.001)$. It was assumed that, in addition to personality dimensions, age, gender, marital status, and academic major can also predict social phobia. Therefore, these variables, in addition to personality dimensions, were entered into the regression equation. The equation was significant $(\mathrm{P}<0.001)$; neuroticism and openness to experience dimensions with beta coefficients of 0.41 and -0.22 , respectively, were effective in social phobia prediction $(\mathrm{P}<0.001)$. Demographic variables were excluded from the regression equation (Table 5).

Table 5: Data summary on the regression coefficients, determination coefficient, and standard error of estimating the impact of personality components on social phobia (stepwise regression method)

\begin{tabular}{cccccc}
\hline Step & Variable & B & SEB & $\beta$ & P-value \\
\hline \multirow{2}{*}{1} & Neuroticism & 0.75 & 0.10 & 0.41 & $<0.001$ \\
\cline { 2 - 6 } & Openness to experience & -0.52 & 0.13 & -0.22 & $<0.001$ \\
\hline
\end{tabular}




\section{Discussion}

In this study, the prevalence of social phobia (moderate, severe, and very severe) in the whole studied population was $58.5 \%$. The prevalence of social phobia (medium-high) in dental students, medical students, and students from other medical specialties was $60.9 \%, 58.9 \%$, $57.4 \%$, respectively. Researchers have reported the prevalence of this disorder in students of different academic majors of Kerman University of Medical Sciences, Iran, as $46 \%$ (16), nursing and midwifery students of Qazvin, Iran, as $28.6 \%$ (18), Malaysian medical students as $56 \%$ (39), English medical students as 8\% (40), and Iraqi medical students as $22 \%$ (41) and $12 \%$ (42), and in Nigerian nursing students as $50 \%$ (43). In this respect, results of the present study were consistent with some cited studies. It seems that differences in culture, measurement instruments, and the statistical population are the reasons for differences in prevalence of social phobia among students from different parts of the world.

In the present study, although average score of social phobia in men was more than in women, this difference was not significant. Momeni, Ghaedi, Chan, and Salina did not find a significant difference between men and women in terms of social phobia and its dimensions (18, 39, 44, 45). However, differences have been reported between men and women in terms of fear in some specific situations such as speeches, presentations, parties, public toilets, and returning a purchased item to the store $(18,39$, $44,45)$. In addition, some studies show that women have higher rate of social anxiety than men in some certain situations $(16,46)$. This difference could be caused by the environment, or cultural and educational differences. It seems that the entry of women into various scientific, economic, and social fields in Iran has affected the gender differences. It is essential to mention that in some studies social anxiety and social phobia have been considered as aligned and sometimes interchangeable while there is a conceptual difference between social anxiety and social phobia. Social anxiety is the fear of negative evaluation in social situations, and social phobia is the evident and constant fear of social situations (5).

In the present study, the average score of extroversion and conscientiousness in male students was higher than female students. In this regard, the results of different studies among different aged groups, cultures, and societies were different. Abedi showed that men and women only had a significant difference in the flexibility dimension (20). Shokri also showed that female students, in comparison with male students, had higher scores in neuroticism and adaptability traits, and male students, compared to female students, had higher scores in extraversion, conscientiousness, and acceptance traits (47). However, Costa found no significant difference between the two sexes in neuroticism and adaptability traits (48). Budav showed that women, compared to men, obtained higher scores in neuroticism and adaptability factors (49). Feingold believes that the results of numerous studies on gender differences have been inconsistent in terms of extraversion and acceptance factors (50). In this regard, cultural, ethnic, and age differences are acceptable in in difference among women and men in terms of personality factors. For example, in this field, Chapman showed that the pattern of gender differences in personality traits can, respectively, be generalized to middle age and old age (51). The results of the present study indicated that dental students' average score of fear (a social phobia dimension) was significantly higher than other groups. Furthermore, the highest prevalence of individuals without social phobia symptoms and individuals suffering from moderate and very severe social phobia were related to students of other medical specialties and the highest prevalence of severe social phobia belonged to dental students. 
Social phobia had relationship direct significant relationship with neuroticism personality dimension and an inverse significant relationship with extroversion, openness to experience, agreeableness, and conscientiousness dimensions. In neurotic characters, unconscious conflicts are severe and painful and defense mechanisms are restrictive to the extent that neurotic symptoms emerge and the individual suffers from anxiety. The results of the study by Marteinsdottir, who investigated personality traits in people suffering from social phobia using the Karolinska Scales of Personality (KSP), showed that people suffering from social phobia are usually sensitive and anxious, have less confidence, and are unable to calm themselves (52). They are usually tired, lack the ability to express their existence, and avoid others. They also fail in social adjustment. Chatterjee also investigated personality traits in people suffering from social phobia using the Temperament and Character Inventory (TCI) (53). His results showed high levels of trauma avoidance and low levels of participation, selfleadership, and exploration in people suffering from social phobia regardless of avoidant personality disorder. These results were similar to that of the present study in which students' personality traits were measured using the NEO PI-R. Hence, it seems that all three scales almost overlap in identifying personality streaks and traits. Val Velzen indicated that high levels of avoidant social phobia, depression symptoms, neuroticism, introversion, and social and professional function disorder are observed in individuals with avoidant personality disorder (33).

The results of his study showed that $85 \%$ of social phobia can be predicted by introversion and depression (33). The results of the study by Reich indicated that social phobia disorder is accompanied by high levels of anxiety in the measurement of various personality dimensions (32). The results of the study by Keuroghlia also showed that marginal personality disorder had a significant relationship with generalized anxiety disorder, social phobia, and post-traumatic stress disorder (PTSD), and significantly predicted the recurrence of social phobia (54). Furthermore, in another study, a strong correlation was observed between neuroticism and social phobia, and a negative correlation was observed between extroversion, and social phobia and social fear (55).

In this study, the researchers tried to arrange for the students to fill the questionnaires when they were not busy with their professional clinical practices; however, it was not possible to fully control this issue. Therefore, it seems that the work environment and time of filling the questionnaire may affect how the questionnaire is completed, and thus, affect the participants' responses.

\section{Conclusion}

It can be concluded that various personality traits have a relation and correlation with social phobia which is a common issue particularly among young people. Therefore, first, families, after undergoing the necessary training on efficient parenting and nurturing methods, should fundamentally and systematically direct children's different personality types toward healthier and unrestricted dimensions in order to reduce future anxiety. Second, when psychologists, teachers, professors, and advisors face such cases, they should behave or provide treatment by considering individuals' different personality traits. Third, at the time of hiring or recruiting people for different jobs, provided that much social interaction is necessary for the desired job or career, individuals should be assigned to jobs by taking their personalities into account. Since, completing the questionnaires coincided with the students' final exams, it seems that this may have somewhat affected the results. 


\section{Acknowledgments}

The authors wish to thank the Vice Chancellor of Research of Rafsanjan University of Medical Sciences, employees of this department, and all students participating in the study.

Conflict of interest: None declared.

\section{Reference}

1. Bitaraf Sh, Shaeeri MR, Hakim Javadi M. Social phobia, parenting styles, and perfectionism. Developmental Psychology: Iranian Psychologists 2010; 7(25):75-82.

2. Heimberg RG. Social phobia: diagnosis, assessment, and treatment. $1^{\text {st }}$ ed. New York City, United States: Guilford Press; 1995.

3. Harvard women's health watch. What to do about social anxiety disorder. The holidays are coming, with lots of convivial occasions--fun for most, but distressing if you suffer from social phobia. Harvard Health Publications, Harvard Medical School, Countway Library of Medicine on the Harvard Medical School Campus in Boston, Massachusetts; 2008 December. Available from: http://www.health.harvard.edu/newsletter_article/ What_to_do_about_social_anxiety_disorder

4. American Psychiatric Association. Diagnostic and statistical manual of mental disorders. $4^{\text {th }}$ ed. Arlington County, Virginia, United States: American Psychiatric Association; 2000.

5. Sadock BJ, Sadock VA, Ruiz P. Kaplan and Sadock's synopsis of psychiatry: behavioral science/clinical psychiatry. $11^{\text {th }}$ ed. Philadelphia, United States: Lippincott Williams and Wilkins; 2014.

6. Chhabra V, Bhatia M, Gupta S, Kumar P, Srivastava Sh. Prevalence of social phobia in school-going adolescents in an urban area. Delhi Psychiatry Journal 2009; 12(1):18-25.

7. Ghazwani JY, Khalil SN, Ahmed RA. Social anxiety disorder in Saudi adolescent boys: Prevalence, subtypes, and parenting style as a risk factor. J Family Community Med 2016; 23(1):25-31.

8. Russell G, Shaw S. A study to investigate the prevalence of social anxiety in a sample of higher education students in the United Kingdom. J Ment Health 2009; 18(3):198-206.

9. Abbo C, Kinyanda E, Kizza RB, Levin J, Ndyanabangi Sh, Stein DJ. Prevalence, comorbidity and predictors of anxiety disorders in children and adolescents in rural north-eastern Uganda. Child Adolesc Psychiatry Ment Health 2013; 7:21. doi: 10.1186/1753-2000-7-21

10. Demir T, Karacetin G, Eralp Demir D, Uysal O. Prevalence and some psychosocial characteristics of social anxiety disorder in an urban population of Turkish children and adolescents. Eur Psychiatry 2013; 28(1):64-9.

11. Landell MG. Social anxiety disorder in swedish adolescents: Prevalence, victimization \& development. Linköping, Sweden: Linköping University; 2010. 86 p. Report No.:1185

12. Baptista CA, Loureiro SR, de Lima Osorio F, Zuardi AW, Magalhaes PV, Kapczinski F, et al. Social phobia in Brazilian university students: prevalence, under-recognition and academic impairment in women. J Affect Disord 2012; 136(3):857-61.

13. Gultekin BK, Dereboy IF. The prevalence of social phobia, and its impact on quality of life, academic achievement, and identity formation in university students. Turk Psikiyatri Derg 2011; 22(3):150-8.

14. Tillfors M, Furmark T. Social phobia in Swedish university students: prevalence, subgroups and avoidant behavior. Soc Psychiatry Psychiatr Epidemiol 2007; 42(1):79-86.

15. Bella TT, Omigbodun OO. Social phobia in Nigerian university students: prevalence, correlates and co-morbidity. Soc Psychiatry Psychiatr Epidemiol 2009; 44(6):458-63.

16. Mazhari Sh, Ekhlaspour M, Banazadeh N. Social phobia and its association with academic performance among student of Kerman University of Medical Sciences, Iran. Journal of Strides in Development of Medical Education 2014; 11(2):227-35.

17. Yaghubi H, Karimi M, Omidi A, Mesbah N, Kahani Sh, Abedi-Qelich-Qeshlaq M. Prevalence of mental disorders and demographic factors that influence the freshmen students of Tehran City Universities of Medical Sciences. J Clin Psychol 2014;6(2):95-104.

18. Momeni M, Zeyghami R, Moradi M, Taherpour M, Sarichloo ME. Prevalence and factors associated with social phobia in nursing and midwifery students. Journal of Health And Care 2015; 17(2):137-45.

19. Bahrami M, Abolghasemi A, Narimani M. Comparing of self-perception and safety measures in students with social anxiety disorder symtoms and normal students. J Sch Psychol 2013; 2(1):62-79. 
20. Sevari K. Predict social competence through loneliness and social phobia. Social Psychology Research 2014; 3(12):37-47.

21. Brook CA, Willoughby T. Social anxiety and alcohol use across the university years: Adaptive and maladaptive groups. Dev Psychol 2016; 52(5):835-45.

22. Kuckertz JM, Strege MV, Amir N. Intolerance for approach of ambiguity in social anxiety disorder. Cogn Emot 2016; 1-8. doi: 10.1080/02699931.2016.1145105

23. Koyuncu A, Ertekin E, Binbay Z, Ozyildirim I, Yuksel C, Tukel R. The clinical impact of mood disorder comorbidity on social anxiety disorder. Compr Psychiatry 2014; 55(2):363-9.

24. Cloutier RM, Blumenthal H, Mischel ER. An examination of social anxiety in marijuana and cigarette use motives among adolescents. Subst Use Misuse 2016; 51(3):408-18.

25. Kawaguchi A, Nemoto K, Nakaaki S, Kawaguchi $\mathrm{T}$, Kan $\mathrm{H}$, Arai N, et al. Insular volume reduction in patients with social anxiety disorder. Front Psychiatry 2016; 7:3. doi: 10.3389/fpsyt.2016.00003

26. Salman A, Kurt E, Topcuoglu V, Demircay Z. Social anxiety and quality of life in vitiligo and acne patients with facial involvement: a crosssectional Controlled Study. Am J Clin Dermatol 2016; 17(3):305-11.

27. Lampe L. Avoidant personality disorder as a social anxiety phenotype: risk factors, associations and treatment. Curr Opin Psychiatry 2016; 29(1):64-9.

28. Torvik FA, Welander-Vatn A, Ystrom E, Knudsen GP, Czajkowski N, Kendler KS, et al. Longitudinal associations between social anxiety disorder and avoidant personality disorder: A twin study. J Abnorm Psychol 2016; 125(1):11424.

29. Berkol TD, Kirli E, Islam S, Pinarbasi R, Ozyildirim I. Comparison of clinical and sociodemographic features of bipolar disorder patients with those of social anxiety disorder patients comorbid with bipolar disorder in Turkey. Saudi Med J 2016; 37(3):309-14.

30. Haghshenas H, Chamani AR, Firoozabadi A. Compare features of personality and mental health of high schools students in gifted and ordinary. The Quarterly Journal of Fundamentals of Mental Health 2005; 8(29-30):57-66.

31. Schultz DP, Schultz SE. Theories of Personality. $9^{\text {th }}$ ed. Boston, United States: wadsworth, Cengage Learning; 2009.
32. Reich J. Avoidant personality disorder and its relationship to social phobia. Curr Psychiatry Rep 2009; 11(1):89-93.

33. Van Velzen CJ, Emmelkamp PM, Scholing A. Generalized social phobia versus avoidant personality disorder: differences in psychopathology, personality traits, and social and occupational functioning. J Anxiety Disord 2000; 14(4):395-411.

34. Eikenaes I, Egeland J, Hummelen B, Wilberg T. Avoidant personality disorder versus social phobia: the significance of childhood neglect. PLoS One 2015; 10(3):e0122846. doi: 10.1371/journal.pone.0122846.

35. Ranta K, La Greca AM, Kaltiala-Heino R, Marttunen M. Social phobia and educational and interpersonal impairments in adolescence: A prospective study. Child Psychiatry Hum Dev 2016; 47(4):665-77.

36. Safarinia M. Social psychology and personality tests. $2^{\text {nd }}$ ed. Tehran: Arjmand Press; 2015.

37. Haghshenas H. Persian version and standardizlJtion of NED personality inventoryrevised. Iranian Journal of Psychiatry and Clinical Psychology 1999; 4(4):34-48.

38. Shakerian A, Fatemi A, Farhadian M. A survey on relationship between personality characteristics and marital satisfaction. Scientific Journal of Kurdistan University of Medical Sciences 2011;16(1):92-9.

39. Salina M, Ng CG, Gill JS, Chin JM ,Chin CJ, Yap WF, et al. Social anxiety problem among medical students in Universiti Malaya Medical Center (Ummc) - a cross-sectional study. Malaysian Journal of Psychiatry Ejournal 2008; 17(2):1-6. Available from: http://www.psychiatrymalaysia.org/article.php?aid $=888$

40. Laidlaw AH. Social anxiety in medical students: Implications for communication skills teaching. Med Teach 2009; 31(7):649-54.

41. Ali SK. 861-Social phobia among medical students. Eur Psychiatry 2013; 28(Supplement 1):1.

42. Alkhafij AZM. Social phobia among Al Qadissya medical student: prevalence, academic performance and response to different treatments. J Fac Med Baghdad 2012; 54(1):33-7.

43. Emmanuel A, Oyedele Emmanuel A, Gimba Solomon M, Gaji Luka D, Kevin Terdi D. Does self-esteem influence social phobia among undergraduate nursing students in Nigeria? International Journal of Medical and Health Research 2015; 1(1):28-33. 
44. Chan VW-Sh. Gender differences associated with social phobia: a developmental perspective. Journal of Undergraduate Life Sciences 2010; $4(1): 82-5$.

45. Ghaedi GH, Tavoli A, Bakhtiari M, Melyani M, Sahragard M. Quality of life in college students with and without social phobia. Soc Indic Res 2010; 97(2):247-56.

46. Abedi Gh, Mohammadi A, Alizadeh A, Hoseini $\mathrm{H}$, Yahyazadeh $\mathrm{O}$. A survey on the relationship between personality factors and sex in students of Mazandaran University of Medical Sciences. Journal of Mazandaran University of Medical Sciences 2013; 23(98):114-22.

47. Shokri O, kadivar P, Farzad V, Sangari AA, Zeinabadi HR, Ghanaee Z, et al. Role of personality factors, learning approaches and thinking styles on academic achievement of college students. Journal of Psychology 2008; 11(4):447-70.

48. Costa PT Jr, Terracciano A, McCrae RR. Gender differences in personality traits across cultures: robust and surprising findings. J Pers Soc Psychol 2001; 81(2):322-31.

49. Budaev SV. Sex differences in the big five personality factors: Testing an evolutionary hypothesis. Pers Individ Dif 1999; 26(5):801-13.

50. Feingold A. Gender differences in personality: a meta-analysis. Psychol Bull 1994; 116(3):429-56.

51. Clark DM. Crozicr WR, Alden LE. The Essential Handbook of Social Anxiety for Clinicians. 1st ed. Hoboken, New Jersey, United States: John Wiley \& Sons, Inc; 2005. Chapter 18, A cognitive perspective on social phobia.

52. Marteinsdottir I, Furmark T, Tillfors $M$, Fredrikson M, Ekselius L. Personality traits in social phobia. Eur psychiatry 2001;16(3):143-50.

53. Chatterjee S, Sunitha TA, Velayudhan A, Khanna S. An investigation into the psychobiology of social phobia: personality domains and serotonergic function. Acta Psychiatr Scand 1997; 95(6):544-50.

54. Keuroghlian AS, Gunderson JG, Pagano ME, Markowitz JC, Ansell EB, Shea T, et al. Interactions of borderline personality disorder and anxiety disorders over 10 years. J Clin Psychiatry 2015; 76(11). doi:10.4088/JCP.14m09748

55. Watson D, Naragon-Gainey K. Personality, emotions, and the emotional disorders. Clin Psychol Sci 2014; 2(4):422-42. 